References:

[1] Collas, A., De Borger, R., Amanova, T., Vande Velde, C.M.L., Baeke, J.K., Dommisse, R., Van Alsenoy, C., Blockhuys, F. (2011). New J. Chem. 35, 649-662.

[2] Collas, A. \& Blockhuys, F. (2011). Acta Cryst. C67, o364-o369.

Keywords: Polar Crystals, Intermolecular Interactions, Unit Cell Dipoles
MS33 Prediction of molecular crystal structures

Chairs: Dr. Jacco van de Streek, Dr. Aurora Cruz-Cabeza

MS33-01

\section{Computational screening for organic drug hydrates}

Doris Braun $^{1}$

1. University of Innsbruck / Institute of Pharmacy, Innsbruck, Austria email: doris.braun@uibk.ac.at

Water has the ability to occupy regular positions in the crystal lattice of other substances and to form molecular compounds, named hydrates. As water is an ever present component of the environment such multi-component crystals can form during many steps of product manufacturing and can have profound effects on the performance of a (drug) product. [1] Hydrate formation is a widespread phenomenon and is known to occur for at least one third of drug molecules. [2] Nevertheless, we are still not able to predict hydrates, their stability and dehydration mechanisms based on the molecular diagram only. With computational crystal structure prediction (CSP) methods being successfully employed for predicting smaller pharmaceutical molecules and even multi-component systems, [3] we tested the potential of CSP for drug hydrates. Stoichiometric and nonstoichiometric hydrate systems were chosen as model compounds for experimental and computational studies.

The experimental screen of two dihydroxybenzoic acid (DHB) isomers indicated hydrate formation for one isomer but not the other. The computationally generated hydrate crystal energy landscapes correctly predicted hydrate formation for 2,4-DHB and its stoichiometric ratios and rationalised why no hydrate was found for 2,5-DHB [4].

For 4-aminoquinaldine (4-AQ) the computational hydrate screening suggested the existence of an unknown monohydrate polymorph, which was then produced and found to be the thermodynamically most stable hydrate form of 4-AQ. [5] Calculating the crystal energy landscapes for hydrates is very complex and computationally (time) demanding, as host and different guest molecules in different stoichiometric ratios have to be considered. Therefore, we tested whether it is possible to derive information about hydrate formation for a $Z^{\prime}=3$ monohydrate and higher hydrates from their $Z^{\prime}=1$ hydrate and anhydrate crystal energy landscapes, respectively. For $o$-phenanthroline the $Z^{\prime}=1$ monohydrate the crystal energy landscape rationalised why the compound forms a very stable $Z^{\prime}=3$ channel hydrate. [6] For the hydrate forming alkaloid brucine and the non-hydrate forming derivative strychnine the evaluation of the anhydrate crystal energy landscapes, in particular the close-packed crystal structures and high-energy open frameworks containing voids of molecular (water) dimensions, allowed us to unravel the diverse solid state behaviour of the two alkaloids at a molecular level. [7]

Thus, modelling at the electronic and atomistic level can provide vital support for unravelling the complexity of or- 
ganic hydrate systems and in particular adds confidence to the interpretation of the experimental data.

Keywords: hydrate, crystal structure prediction
MS33-02

\section{Computation-led discovery of functional molecular materials}

\author{
Graeme Day ${ }^{1}$ \\ 1. Chemistry, Southampton, United Kingdom \\ email: g.m.day@soton.ac.uk
}

The design of molecular crystals with targeted properties is the goal of crystal engineering. However, our predictive understanding of how a crystal's properties relate to its structure, and how crystal structure in turn relates to molecular structure, are not yet sufficiently reliable to confidently design functional materials. One reason for this is that the crystal structure adopted by a molecule is rarely determined by a single, predictable structure-directing interaction, but typically results from a balance of many relatively weak intermolecular interactions. It is, therefore, common for a molecule to have many nearly equi-energetic possible crystal structures, with the best structure (the global lattice energy minimum) favoured by only a few $\mathrm{kJ} \mathrm{mol}^{-1}$ or less over alternative structures that might have very different physical properties. This existence of competing low energy crystal structures is related to the prevalence of polymorphism in molecular crystals, as well as the observation that small changes to chemical structure can lead to dramatic changes in crystal packing.

Computational methods for crystal structure prediction (CSP) have been developed to help anticipate the crystal structure that a molecule will form. These methods are based on a global search of the lattice energy surface and a ranking of local energy minima according to their calculated relative stabilities. Each of the crystal structures in the resulting ensemble encodes a set of properties, many of which are calculable using computer simulations. This talk will discuss how the set of predicted structures, their calculated energies and simulated properties, which we present as an energy-structure-function (ESF) map, can be used to guide experimental programmes for materials discovery. The ESF mapping approach will be illustrated with its use in the discovery of unprecedented porous molecular crystals[1] and its application to organic semiconductors[2,3].

References:

[1]. A. Pulido et al, Nature 2017, 543, 657.

[2]. J. E. Campbell, J. Yang and G. M. Day, J. Mat. Chem. C 2017, 5,7574 .

[3] F. Musil, S. De, J. Yang, J. E. Campbell, G. M. Day and M. Ceriotti. Chemical Science, 9, 1289-1300 (2018)

Keywords: crystal structure prediction, materials discovery, functional materials 